\title{
Unusual metal abundances in a pair of damped Lyman alpha systems at $z \sim 2^{\star}$
}

\author{
S. L. Ellison ${ }^{1}$ and S. Lopez ${ }^{2}$ \\ 1 European Southern Observatory, Casilla 19001, Santiago 19, Chile \\ e-mail: sellison@eso.org \\ 2 Departamento de Astronomía, Universidad de Chile, Casilla 36-D, Santiago, Chile \\ e-mail: slopez@das.uchile.cl
}

Received 4 September 2001 / Accepted 9 October 2001

\begin{abstract}
We present high resolution spectroscopic observations of two neighbouring damped Lyman $\alpha$ systems (DLAs) along the same line of sight towards B2314-409. Due to their separation $\left(v \sim 2000 \mathrm{~km} \mathrm{~s}^{-1}\right)$ and the high spectral resolution of the data, it is possible to fit not only the weak metal transitions, but also the separate H I absorption profiles. This has permitted, for the first time, a detailed study of metal abundances in two neighbouring galaxy-scale absorbers. The two DLAs have $z_{\mathrm{abs}}=1.8573$ and 1.8745 and have column densities $\log N(\mathrm{H} \mathrm{I})=20.9 \pm 0.1$ and $20.1 \pm 0.2$ respectively. We have determined abundances for a range of chemical elements, and find that both absorbers towards B2314-409 have low $\alpha / F e-p e a k$ abundances compared with other known DLAs. This indicates that not only has the recent star formation history of these absorbers been relatively passive, but that the group environment, or some other external factor, may have influenced this.
\end{abstract}

Key words. quasars: general - quasars: absorption lines - quasars: individual: B2314-409 - galaxies: evolution galaxies: clusters: general

\section{Introduction}

Since the discovery that intermediate redshift $(z \sim 0.3)$ clusters exhibit a relative over-abundance of blue member galaxies compared with the local population (Butcher \& Oemler 1987), extensive work has investigated the evolution of the cluster environment and compared it with the field population (e.g. Poggianti et al. 1999 and references therein). From these recent spectroscopic studies of $z \sim 0.4$ clusters, it has been established that star formation is generally suppressed in these rich environments, but that post-starburst ( $\mathrm{E}+\mathrm{A}$ ) galaxies make up $\sim 20 \%$ of the cluster population (Dressler et al. 1999). In addition, there appears to be a radial star formation rate gradient in clusters that is independent of the morphology-density relation, such that galaxies with the most recent star formation episodes occur farther out (Balogh et al. 1999). Once accreted into the cluster, active star formation seems to be swiftly quenched (Dressler et al. 1999) and continues at a relatively low rate (e.g. Couch et al. 2001).

Send offprint requests to: S. Ellison,

e-mail: sellison@eso.org

* The work presented here is based on data obtained with UVES at the VLT, program 267.A-5707.
However, these informative surveys have not been extended beyond $z \sim 1$ due to the lack of good quality spectroscopic data at these redshifts, although wide field surveys at X-ray, optical and near-IR wavelengths have detected clusters out to $z \sim 1.3$ (e.g. Rosati et al. 1998). At earlier epochs, the study of Lyman break galaxies (LBGs) has permitted the discovery of large galaxy overdensities at $z \sim 3$ (Steidel et al. 1998). However, it is important to bear in mind that being such biased tracers of matter, LBGs are very different from typical cluster galaxies at low redshifts. We are left, therefore, with a significant gap in our knowledge of groups and clusters of galaxies between $1 \lesssim z \lesssim 3$. In particular, this leaves open many issues involving the early evolution of galaxy groups. For example, at what stage does the environment start to affect the star formation of the individual galaxies and is the activity boosted prior to being truncated?

One of the most promising techniques for detecting representative galaxies at $z \gtrsim 1.5$ is using QSO absorption lines, although the possibilities for studying clusters of absorbing galaxies is more limited. Nonetheless, some observations of high column density absorbers, in particular damped Lyman alpha systems (DLAs), along multiple lines of sight have been supplemented with Lyman break and narrow band Lyman $\alpha$ imaging to show that 


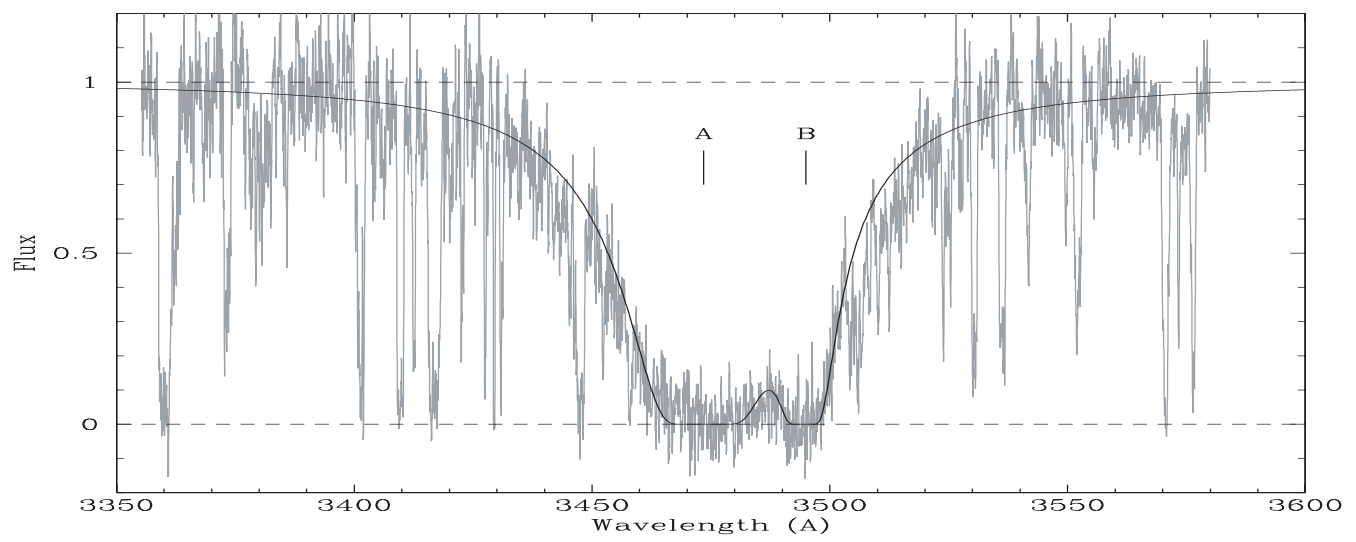

Fig. 1. Profile fit to the double DLA towards B2314-409. The two absorbers have $\log N(\mathrm{H} \mathrm{I})=20.9$ at $z_{\text {abs }}=1.8573$ (DLA A) and $\log N(\mathrm{H} \mathrm{I})=20.1$ at $z_{\mathrm{abs}}=1.8745(\mathrm{DLA} \mathrm{B})$.

DLAs can reside in galaxy concentrations out to $z \sim 3.5$ (e.g. Francis \& Hewett 1993; Francis et al. 1997; Ellison et al. 2001), although there is currently no evidence that DLAs cluster strongly with LBGs (Gawiser et al. 2001). In addition, the presence of metal line profiles with components separated by many hundreds of $\mathrm{km} \mathrm{s}^{-1}$ provides further evidence that DLAs may have near neighbours (e.g. Pettini et al. 1999; Prochaska \& Wolfe 1999). However, due to the difficulty in determining the $N(\mathrm{H} \mathrm{I})$ for individual components, it has so far not been possible to perform full abundance analyses of these proximate absorbers.

Here we present high resolution spectroscopic observations of a DLA pair (i.e. two proximate absorbers in the same line of sight) for which the abundances of the individual galaxies can be studied in detail (Sect. 2) ${ }^{1}$. In addition to determining column densities for several metal line transitions, the UVES spectra presented here have permitted us to resolve the two Lyman $\alpha$ lines, allowing us to determine values for $N(\mathrm{H} \mathrm{I})$ and therefore calculate abundances (Sect. 3). Various explanations for the unusual abundances exhibited by this DLA pair are discussed (Sect. 4), including dust and photo-ionization. Since the chance alignment of two DLAs in single sightline is small, we also consider the possibility that this double absorber is part of some galaxy structure at $z \sim 2$ and therefore whether their unusual abundances may be attributed to their environment.

\section{Observations and data reduction}

Discovered as part of the CORALS survey for DLAs, the absorbers towards B2314-409 were identified by Ellison et al. (2002) as a potential DLA pair, based on the asymmetric H I profile and presence of metals separated by $\sim 2000 \mathrm{~km} \mathrm{~s}^{-1}$. Four hours of Director's Discretionary time with UVES were granted to observe B2314-409 at high spectral resolution in order to determine more accurately

\footnotetext{
${ }^{1}$ We note that according to the historical definition, only one of these absorbers is technically a DLA, despite the presence of clear, broad damping wings.
}

the DLA profile and metal abundances. These observations were carried out on August 12001 with the dichroic $390+564$ setting on UVES, the echelle spectrograph at the VLT, providing almost continuous wavelength coverage from 3300 to $6650 \AA$. The data were reduced using the UVES pipeline, a detailed description of which can be found in Ballester et al. (2000). Once extracted, the individual frames $(R \sim 43000)$ were corrected to a vacuum heliocentric scale and combined with a weight proportional to their $\mathrm{S} / \mathrm{N}$ which varied between 10 and 25 . Finally, regions of the spectrum with absorption lines were normalised by dividing through by a spline function fitted through the QSO continuum.

The normalised section of the spectrum containing the DLA absorbers is shown in Fig. 1 together with a profile fit for the two systems. The redshifts and H I column densities determined for the absorbers are $z_{\mathrm{abs}}=1.8573$ and $\log N(\mathrm{H} \mathrm{I})=20.9 \pm 0.1$ for DLA A and $z_{\mathrm{abs}}=1.8745$ and $\log N(\mathrm{H} \mathrm{I})=20.1 \pm 0.2$ for DLA B, corresponding to a proper separation of $11 \mathrm{Mpc} h^{-1}\left(H_{0}=70 \mathrm{~km} \mathrm{~s}^{-1} \mathrm{Mpc}^{-1}\right.$, $\left.\Omega_{M}=0.3, \Omega_{\Lambda}=0.7\right)$. These fits are constrained mostly by the shape of the damping wings, although smoothing the data enhances the flux recovery between the troughs that is already discernible in Fig. 1. A variety of metal lines associated with these DLAs was identified and fitted with $\mathrm{VPFIT}^{2}$ to determine abundances. As is common practice, we fixed the $b$-values and redshifts of each absorption component, allowing only the column density to vary. Despite the fact that the same transitions were covered for both DLAs, the difference in their metallicities and $\mathrm{H}$ I column densities means that different transitions had to be used to determine abundances in each case. Typically, the transitions that were saturated in DLA A provided good profile fits for DLA B, whilst the useful transitions fitted in DLA A were not detected in DLA B. Table 1 details the component parameters determined for the both DLAs (shown in Figs. 2 and 3) and Table 2 lists the total column densities and abundances.

\footnotetext{
${ }^{2}$ Available at http://www.ast.cam.ac.uk/ ${ }^{\sim} \mathrm{rfc} / \mathrm{vpfit.html}$
} 
S. L. Ellison and S. Lopez: Unusual metal abundances in a pair of damped Lyman alpha systems at $z \sim 2$
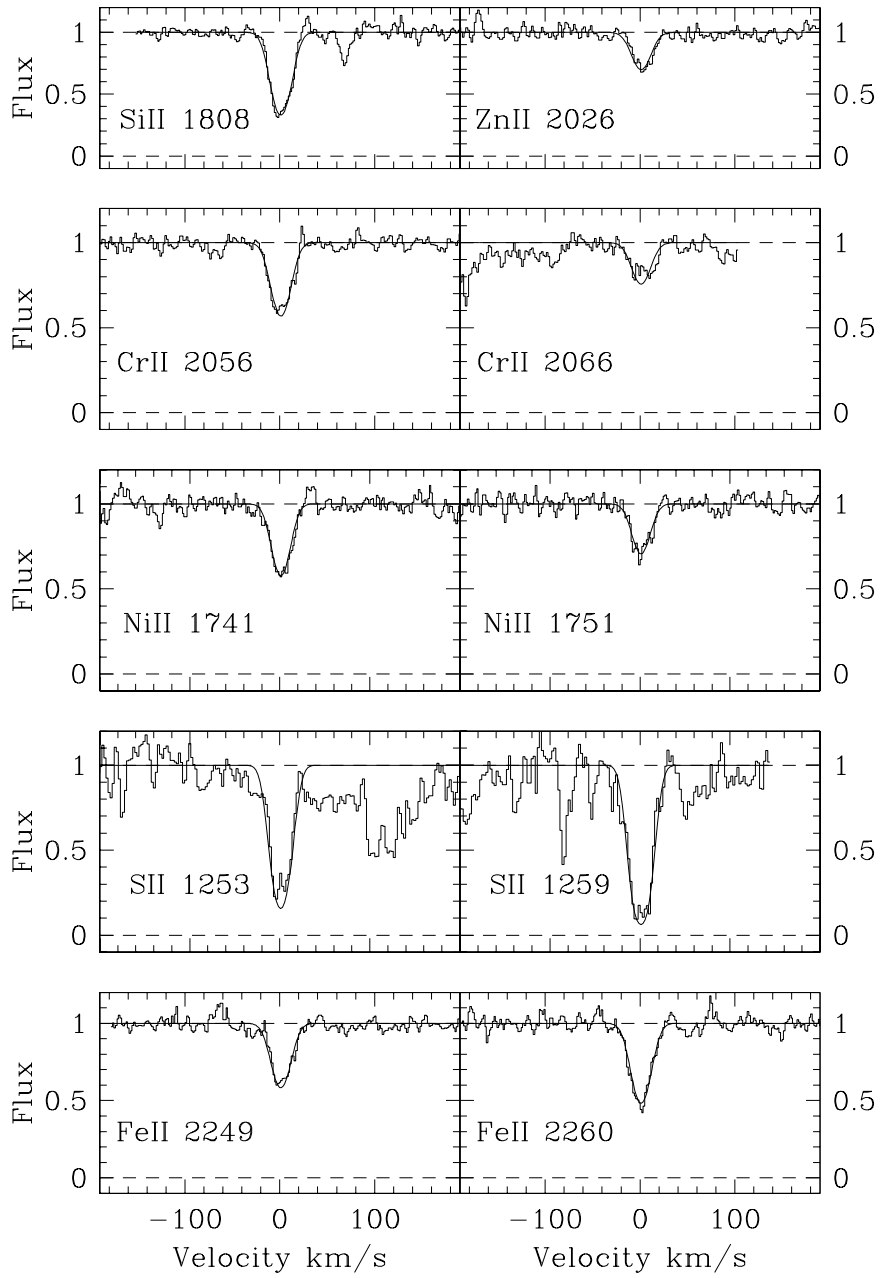

Fig. 2. Unsaturated metal lines associated with DLA A with Voigt profile fits overlaid. The velocity scale on each panel is relative to $z_{\text {abs }}=1.8573$.

\section{Abundances and kinematics}

The abundances determined from Voigt profile fitting for both DLAs are somewhat unusual, as we discuss in more detail below. An interesting possibility is that these absorbers may be part of a large structure, for example a proto-cluster or galaxy filament. If so, the main environmentally driven effects we may expect to witness will most likely impact upon the gas kinematics and chemical abundances of the two systems.

The overall metallicity of both DLAs is typical of those measured at this redshift (Pettini et al. 1999), indicating that there is no particular evidence of extended periods of either highly enhanced or suppressed star formation over the span of each galaxy's star-forming life. However, we note that in documented DLAs there is a large observed spread of metallicities at a given redshift, so that to notice a marked difference in $[\mathrm{Zn} / \mathrm{H}]$ or $[\mathrm{Fe} / \mathrm{H}]$ would require a very pronounced effect. Next we consider the abundances of $\alpha$ elements which, when compared with Fe-peak elements, provide clues to the history of star formation in the galaxy. In DLA A, both [Si/Fe] and $[\mathrm{S} / \mathrm{Zn}]$ are roughly solar (see Table 2), Zn being the usual Fe-peak element of

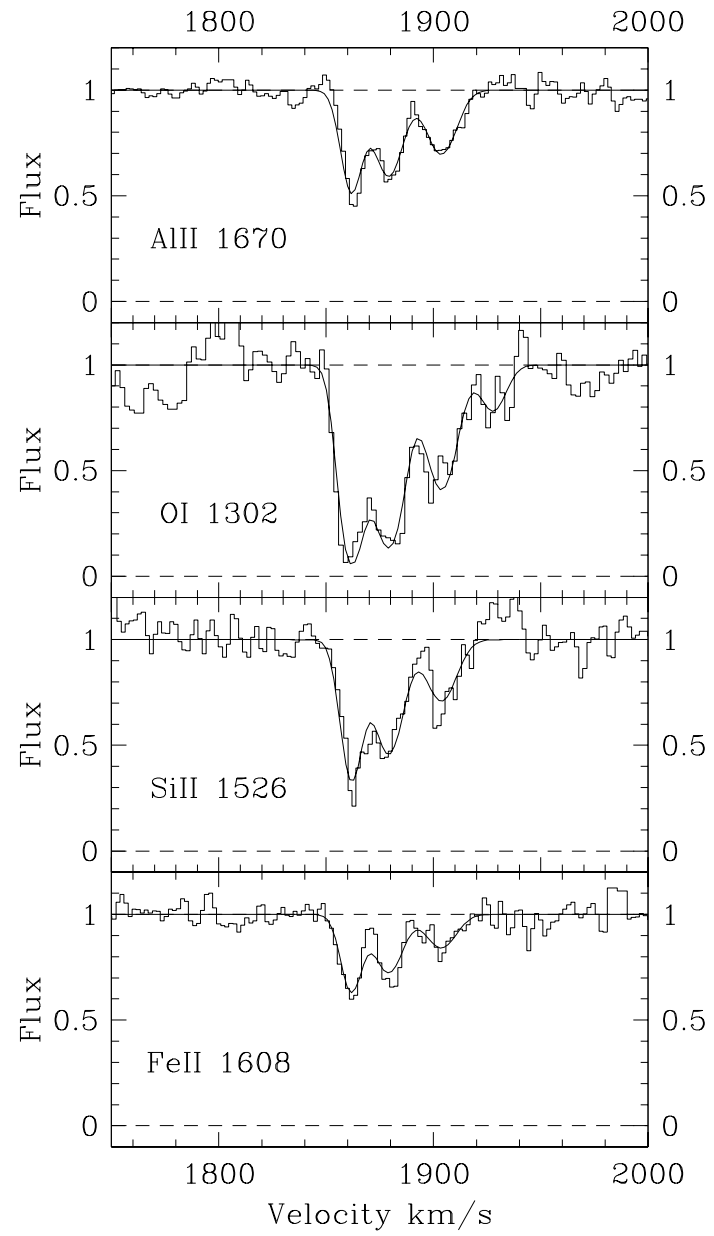

Fig. 3. Unsaturated metal lines associated with DLA B with Voigt profile fits overlaid. The velocity scale on each panel is relative to $z_{\mathrm{abs}}=1.8573$.

choice because of its non-refractory nature. However, since there are very few $[\mathrm{S} / \mathrm{Zn}]$ measurements, in Fig. 4 we plot $[\mathrm{S} / \mathrm{Fe}]$ in order to facilitate a useful comparison with literature values. Combined with the lower limit $[\mathrm{S} / \mathrm{Fe}]<0.15$ in DLA B, Fig. 4 reveals that both absorbers have relatively low $\mathrm{S} / \mathrm{Fe}$ ratios compared with other known DLAs. Note that although the fit to Fe II $\lambda 1608$ in DLA B appears somewhat poor (due to fixing the $b$-values), allowing a completely free fit to the data results in only a 0.01 dex change in $N(\mathrm{Fe})$. The abundance ratios plotted in Fig. 4 relative to Fe will all require some dust correction, which will be different from system to system (see next section). However, we nevertheless note that the $[\mathrm{Si} / \mathrm{Fe}]$ ratios for both DLA A and B are lower than any in the UCSD DLA database (Prochaska et al. 2001) and the large compilation of $\mathrm{Lu}$ et al. $(1996)^{3}$. Furthermore, for DLA B we determine a very low $[\mathrm{O} / \mathrm{Fe}]=-0.40$, which would be further reduced if there was some correction to be made to Fe due to dust. In reality, however, $[\mathrm{O} / \mathrm{Fe}]=-0.40$ is probably a

\footnotetext{
${ }^{3}$ Since several of the $\mathrm{Fe}$ abundances quoted by $\mathrm{Lu}$ et al. (1996) are based on interpolation between upper and lower limits, we only plot the firm detection in Fig. 4, taking into account the required corrections for updated $f$-values.
} 
Table 1. Voigt profile fit parameters for DLAs A and B towards Q2314-409.

\begin{tabular}{|c|c|c|c|c|c|c|c|c|c|c|}
\hline \multirow[t]{2}{*}{ Cloud } & \multirow[t]{2}{*}{ Redshift } & \multirow[t]{2}{*}{$b$} & \multicolumn{8}{|c|}{$\log _{10} N(\mathrm{X})$} \\
\hline & & & $\mathrm{Fe}$ II & Zn II & Cr II & $\mathrm{Al} \mathrm{II}$ & Si II & O I & $\mathrm{Ni} \mathrm{II}$ & S II \\
\hline \multicolumn{11}{|c|}{ DLA A } \\
\hline 1 & 1.857311 & 12.98 & 15.08 & 12.52 & 13.38 & $\ldots$ & 15.41 & $\ldots$ & 13.84 & 15.10 \\
\hline \multicolumn{11}{|l|}{ DLA B } \\
\hline 1 & 1.875032 & 5.02 & 13.34 & ... & $\ldots$ & 11.99 & 13.43 & 14.41 & ... & $\ldots$ \\
\hline 2 & 1.875197 & 7.74 & 13.31 & $\ldots$ & $\ldots$ & 12.01 & 13.38 & 14.28 & $\ldots$ & $\ldots$ \\
\hline 3 & 1.875431 & 8.42 & 13.07 & $\ldots$ & $\ldots$ & 11.87 & 13.05 & 13.95 & $\ldots$ & $\ldots$ \\
\hline 4 & 1.875661 & 6.82 & $\ldots$ & $\ldots$ & $\ldots$ & $\ldots$ & $\ldots$ & 13.32 & $\ldots$ & $\ldots$ \\
\hline
\end{tabular}

Table 2. Abundance measurements (and $3 \sigma$ upper limits) for DLAs A and B towards B2314-409.

\begin{tabular}{ccccc}
\hline \hline & \multicolumn{3}{c}{ DLA A } & \multicolumn{2}{c}{ DLA B } \\
$\mathrm{X}$ & $N(\mathrm{X})$ & {$[\mathrm{X} / \mathrm{H}]$} & $N(\mathrm{X})$ & {$[\mathrm{X} / \mathrm{H}]$} \\
\hline $\mathrm{Fe}$ & $15.08 \pm 0.10$ & $-1.33 \pm 0.14$ & $13.73 \pm 0.1$ & $-1.88 \pm 0.24$ \\
$\mathrm{Si}$ & $15.41 \pm 0.10$ & $-1.44 \pm 0.14$ & $13.79 \pm 0.10$ & $-1.86 \pm 0.22$ \\
$\mathrm{Cr}$ & $13.38 \pm 0.08$ & $-1.20 \pm 0.13$ & $<12.17$ & $<-1.61$ \\
$\mathrm{Zn}$ & $12.52 \pm 0.10$ & $-1.02 \pm 0.14$ & $<11.56$ & $<-1.19$ \\
$\mathrm{Ni}$ & $13.84 \pm 0.08$ & $-1.31 \pm 0.13$ & $<12.70$ & $<-1.65$ \\
$\mathrm{~S}$ & $15.10 \pm 0.15$ & $-1.07 \pm 0.18$ & $<13.64$ & $<-1.73$ \\
$\mathrm{O}$ & $\ldots$ & $\ldots$ & $14.75 \pm 0.12$ & $-2.28 \pm 0.22^{a}$ \\
$\mathrm{Al}$ & $\ldots$ & $\ldots$ & $12.44 \pm 0.08$ & $-2.14 \pm 0.22$ \\
\hline
\end{tabular}

${ }^{a}$ Probably a lower limit due to mild saturation of the O line.

lower limit because of mild saturation of the O line (see next section). Overall, both DLAs exhibit relatively low $\alpha /$ Fe-peak abundances, although not excessively so, given the uncertainties.

Although DLA A appears to be a single component from unsaturated lines, stronger transitions such as Si II $\lambda 1526$ and Al II $\lambda 1670$ reveal this system to have a somewhat more complicated multi-component structure. In fact, both DLAs have absorption profiles that extend over approximately $100 \mathrm{~km} \mathrm{~s}^{-1}$, a velocity not atypical compared with other damped systems (Prochaska \& Wolfe 2001). It therefore appears that the interstellar gas has not undergone significant disruption. The few observations of other absorbers in high redshift galaxy groups provide mixed results with regards to kinematics. Q0201+1120 has a velocity spread of $300 \mathrm{~km} \mathrm{~s}^{-1}$ consisting of many components (Ellison et al. 2001). Similarly, the DLA at $z_{\text {abs }}=2.38$ towards B2138-4427 has components over $200 \mathrm{~km} \mathrm{~s}^{-1}$ (C. Ledoux, private communication), but the possible LLS in the same group towards B2139-4434 has only a $\sim 60 \mathrm{~km} \mathrm{~s}^{-1}$ spread (V. D'Odorico, private communication).

\section{Possible explanations for unusual abundance ratios}

The results of Fig. 4 are striking. It is particularly noteworthy that both DLA A and B exhibit unusually low $\alpha /$ Fe-peak ratios, as does the DLA in the group towards
Q0201+1120 (Ellison et al. 2001). However, before drawing the conclusion that this is evidence that the environment has a significant impact on star formation histories at $z \sim 2$, we explore other factors that may affect our abundance determinations.

We first consider the possibility of partial photoionization. This is unlikely to be an issue for the relatively high $N(\mathrm{H} \mathrm{I})$ DLA A, but may have an effect on DLA B. Evidence that low $N(\mathrm{H} \mathrm{I})$ DLAs and sub-DLAs may be increasingly affected by photo-ionization comes from the increasing fraction of $N(\mathrm{Al} \mathrm{III}) / N(\mathrm{Al}$ II) found by Vladilo et al. (2001). However, the upper limit we determine for the ratio of $N(\mathrm{Al} \mathrm{III}) / N(\mathrm{Al} \mathrm{II})<-0.91$ is significantly lower than that predicted for a $\log N(\mathrm{H} \mathrm{I})=20.1$ from the trend found by Vladilo et al. (2001). This provides direct evidence that photo-ionization does not have a significant impact on these abundance determinations. Specifically, in the case of $[\mathrm{O} / \mathrm{Fe}]$, the calculations of Vladilo et al. (2001) show that Fe II is converted to Fe III as effectively as $\mathrm{O}$ I is ionized to O II, so that partial ionization is highly unlikely to be the reason for the low $\mathrm{O}$ abundance. In fact, according to Vladilo et al. (2001), a ionization correction would have a greater effect on $[\mathrm{Si} / \mathrm{Fe}]$ pushing it to a lower value and enhancing the effect seen in Fig. 4.

Next, we consider the effects of saturation. As mentioned in the previous section, it is plausible that the O I $\lambda 1302$ transition is mildly saturated, requiring an upward revision of $N(\mathrm{O})$. In fact, it is possible to achieve a fit with the same $\chi^{2}$ statistic if $N(\mathrm{O})$ is increased by $0.4 \mathrm{dex}$ and the $b$-values allowed to vary freely. A reduction of the $b$-values by only $\sim 1.0 \mathrm{~km} \mathrm{~s}^{-1}$ in the two strongest components is sufficient to achieve this. The additional corollary of increasing $N(\mathrm{O})$ is to bring the $\mathrm{O} / \mathrm{Si}$ ratio in closer agreement with solar values. On the other hand, the high spectral resolution of these data make the possibility of "hidden" saturation in other absorption lines highly unlikely. Indeed, the excellent agreement between column densities of $\mathrm{Fe}$ and $\mathrm{Ni}$ determined by using transitions with different oscillator strengths shows that there is no hidden saturation effect for the other absorption lines used here.

Finally, we consider the possibility of atypical ammounts of dust depletion. The low $[\mathrm{S} / \mathrm{Fe}]$ abundance shown in Fig. 4 may be due to anomalously low amounts of dust in these DLAs, as supported by the low $[\mathrm{Zn} / \mathrm{Cr}]$ ratio 

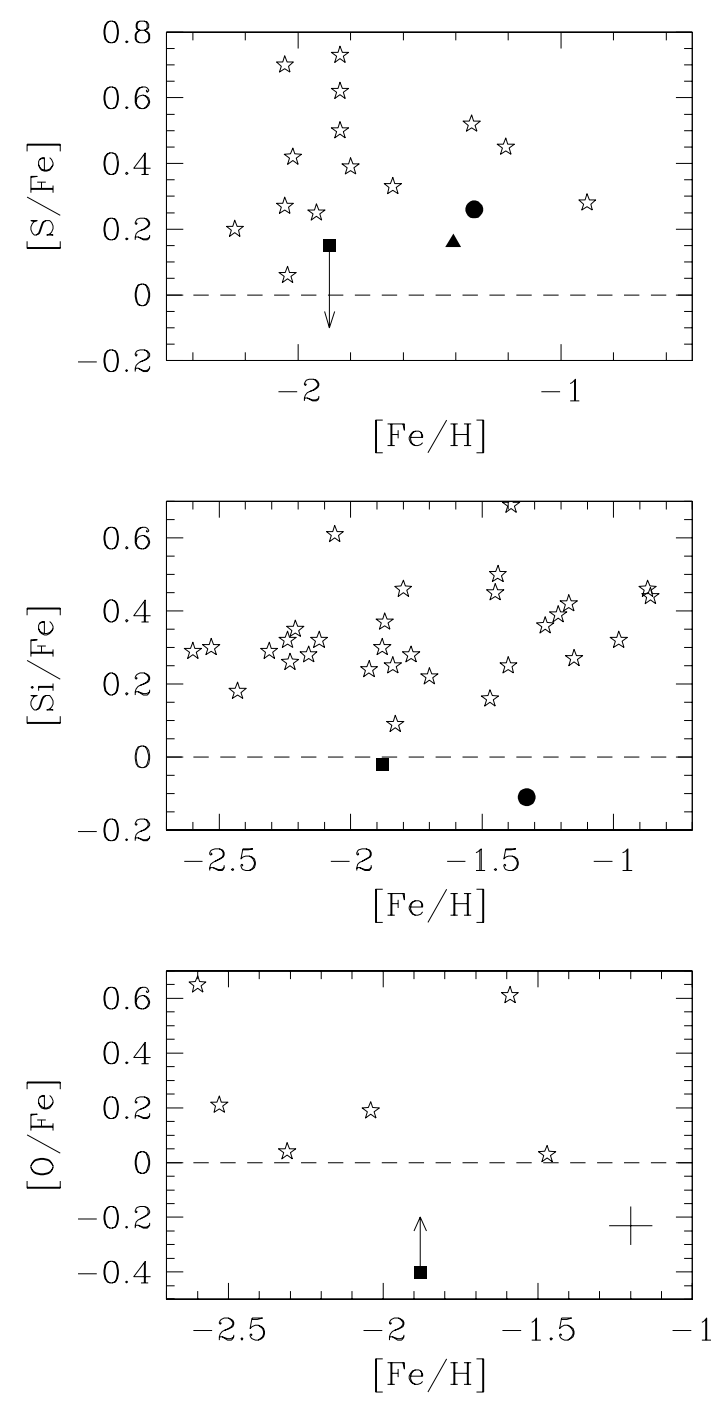

Fig. 4. Relative abundances of $\alpha /$ Fe-peak elements. In all panels, open stars are literature values (Molaro et al. 2001; Outram et al. 1999; Lu et al. 1998; Ge et al. 2001; Pettini et al., in prep; Lu et al. 1996; Centurión et al. 2000; Prochaska et al. 2001), the filled triangle is for the DLA found towards Q0201+1220 in a galaxy group at $z \sim 3.4$ (Ellison et al. 2001), filled circles and squares represent values determined for DLAs A and B respectively. The typical error bar is shown in the lower right corner of the bottom panel.

in DLA A. However, dust is unlikely to be the explanation for the low $[\mathrm{Si} / \mathrm{Fe}]$ ratios, since this would require that $\mathrm{Si}$ be more depleted than Fe, contrary to what is observed locally (Savage \& Sembach 1996). The middle panel of Fig. 4 is therefore the most convincing evidence that the intrinsic abundances of this double DLA are anomalous compared with other systems in the literature.

\section{Discussion}

We have presented high resolution spectroscopic observations of a pair of DLAs separated by $\sim 2000 \mathrm{~km} \mathrm{~s}^{-1}$ $\left(\sim 11 \mathrm{Mpc} h_{70}^{-1}\right)$ at $z_{\mathrm{abs}} \sim 2$ and propose the possibility that these absorbers may form part of an extended structure. Absorbing structures of similar size at high $z$ have previously been inferred from multiple lines of sight, for example the posited super-cluster at $z \sim 2$ towards Tol 1037-2704 (Sargent \& Steidel 1987). However, this is the first time that the abundances of two proximate DLAs in a single line of sight have been studied in detail. One other multiple system, a triple DLA towards CTQ 247 (Lopez et al. 2000), has been recorded in the literature and a program has been initiated to study its abundances.

Although we have found that the metallicity of both DLAs is typical of that measured in other such systems at the same redshift, there is evidence from their unusual abundance ratios that these absorbers may have experienced somewhat different star formation histories than most other documented DLAs. This evidence comes from the generally low $\alpha /$ Fe-peak abundances of both DLAs, and the DLA in the group towards Q0201+1120 (Ellison et al. 2001), suggestive that their global environment may have played some role in their evolution. Despite the possible effects of saturation (in the case of O I $\lambda 1302$ ) and atypically low amounts of dust (which may explain $[\mathrm{S} / \mathrm{Fe}])$, the low $[\mathrm{Si} / \mathrm{Fe}]$ compared to the large number of literature values remains convincing evidence that star formation history may be responsible for these ratios. Such low $\alpha /$ Fe-peak abundances are usually interpreted as the signatures of low star formation rates, or of an ISM enriched by an early generation of stars that is now evolving quiescently. Therefore, the abundances observed here are reminiscent of the truncated activity observed in lower $z$ clusters.

At intermediate redshifts $(z \sim 0.5)$, observations indicate that star formation is suppressed in rich cluster galaxies (possibly after an initial burst of enhanced activity) by processes such as ram pressure and tidal stripping (Couch et al. 2001 and references therein). Just as this process manifests itself as an excess of post-starburst $(\mathrm{E}+\mathrm{A})$ galaxies with no strong emission lines but strong Balmer absorption, so we may expect to see the chemical signature of star formation truncation. However, it would be somewhat surprising if such processes already have such an effect on star formation at $z \sim 2$ where environments are relatively poor and canonical rich clusters have yet to form. In addition, although the kinematics of these DLAs, determined from the unsaturated metal lines, extend over $\sim 100 \mathrm{~km} \mathrm{~s}^{-1}$, this is not atypical of the range exhibited by other DLAs, i.e. there is no evidence for significant disturbance of the ISM.

Follow-up observations of this field, by either multicolour (to determine photometric redshifts) or narrow band Lyman $\alpha$ imaging are clearly of great interest to confirm the richness of the environment around these DLAs. In addition, it is important to identify other multiple DLAs and follow them up with high resolution spectroscopy in order to ascertain whether DLAs in groups exhibit distinct abundances from their "field" counterparts.

Acknowledgements. S.L. acknowledges financial support by FONDECYT grant No. 3000001 and by the Deutsche 
Zentralstelle für Arbeitsvermittlung. We are grateful to Marcin Sawicki for useful discussions and to Max Pettini and Jason Prochaska (the referee) for comments and suggestions that have helped to improve this work.

\section{References}

Ballester, P., Modigliani, A., Boitquin, O., et al. 2000, ESO Messenger, 101, 31

Balogh, M., Morris, S., Yee, H. K. C., Carlberg, R. G., \& Ellingson, E. 1999, ApJ, 527, 54

Butcher, H., \& Oemler, A . 1978, ApJ, 226, 559

Centurión, M., Bonifacio, P., Molaro, P., \& Vladilo, G. 2000, ApJ, 536, 540

Couch, W., Balogh, M., Bower, R., et al. 2001, ApJ., 549, 820

Dressler, A., Smail, I., Poggianti, B., et al. 1999, ApJS, 122, 51

Ellison, S. L., Pettini, M., Steidel, C. C., \& Shapley, A. 2001, ApJ, 549, 770

Ellison, S. L., Yan, L., Hook, I., et al. 2001, A\&A, accepted [astro-ph/0109205]

Francis, P., \& Hewett, P. 1993, AJ, 105, 1633

Francis, P., Woodgate, B., \& Danks, A. 1997, ApJ, 482, 25

Gawiser, E., Wolfe, A., Prochaska, J., et al. 2001, ApJ, in press [astro-ph/0103387]

Ge, J., Bechtold, J., \& Kulkarni, V. 2001, ApJL, 547, 1
Lopez, S., Maza, J., Masegosa, J., \& Marquez, I. 2000, A\&A, 366,387

Lu, L., Sargent, W. L. W., \& Barlow, T. A. 1998, AJ, 115, 55

Lu, L., Sargent, W. L. W., Barlow, T. A., Churchill, C. W., \& Vogt, S. S. 1996, ApJS, 107, 475

Molaro, P., Levshakov, S., D'Odorico, S., Bonifacio, P., \& Centurión, M., 2001, ApJ, 2000, 549, 90

Outram, P., Chaffee, F., \& Carswell, R., 1999, MNRAS, 310, 289

Pettini, M., Ellison, S. L., Steidel, C. C., \& Bowen, D. V. 1999, ApJ, 510, 576

Poggianti, B., Smail, I., Dressler, A., et al. 1999, ApJ, 518, 576

Prochaska, J.X., \& Wolfe, A.M. 1999, ApJS, 121, 369

Prochaska, J. X., \& Wolfe, A. M. 2001, ApJ, submitted [astro-ph/0108154]

Prochaska, J. X., Wolfe, A., Tytler, D., et al. 2001, ApJ, in press

Rosati, P., della Ceca, R., Norman, C., \& Giacconi, R., 1998 ApJL, 492, L21

Sargent, W., \& Steidel, C., ApJ 1987, 322, 142

Savage, B. D., \& Sembach, K. R. 1996, ARA\&A, 34, 279

Steidel, C. C., Adelberger, K. L., Dickinson, M., et al. 1998, ApJ, 492, 428

Vladilo, G., Centurion, M., Bonifacio, P., \& Howk, J. C. 2001, ApJ, 557, 1007 